\title{
Giovani e tecnologie: tra nativi digitali e competenze effettive
}

\section{Sandra Zampieri, Luca Botturi e Spartaco Calvo}

Il confronto generazionale nell'ambito delle tecnologie digitali è stato fortemente segnato dal concetto di "nativo digitale», che contrappone le nuove generazioni ai più anziani «immigrati digitali». Diversi studi confutano queste tesi, o quantomeno le mettono in discussione, anche se il dibattito è ancora aperto. L'indagine ICILS offre dati solidi per approfondire il rapporto tra giovani e tecnologie, in particolare contestualizzandolo in un contesto geografico e sociale puntuale nel quale le istituzioni scolastiche non hanno finora assunto un ruolo importante e definito. Questo studio cerca di rispondere alle seguenti domande: Quali caratteristiche del sistema educativo favoriscono lo sviluppo di competenze digitali? Esiste una relazione tra età di inizio dell'uso delle tecnologie, frequenza d'uso e tipologia d'uso e le competenze digitali nei diversi ambiti? In conclusione si cerca di aprire la riflessione al ruolo della scuola e delle politiche educative nell'uso dei dispositivi digitali e di internet da parte degli allievi.

\section{Introduzione: nativi digitali tra ricerca e scuola}

La rapida e pervasiva diffusione delle tecnologie digitali in tutti gli ambiti personale, professionale, industriale, e istituzionale - è allo stesso tempo un dato di fatto e un elemento nella strategia di alcuni Stati, tra cui la Svizzera (Bundesamt für Kommunikation $[\mathrm{AKOM}], 2016)$. Questa evoluzione ha portato alla ribalta il grande tema dello sviluppo di competenze digitali nei giovani, come indicato anche dal Rapporto sulla digitalizzazione di recente pubblicato dalla Segreteria di stato per la Formazione, la Ricerca e l'innovazione (Segreteria di Stato per la formazione, la ricerca e l'innovazione [SEFRI], 2017). Il loro inserimento integrale come cittadini e come professionisti richiederà sempre maggiori competenze digitali, e questo ha portato alla definizione di nuove sfide per i sistemi educativi (OECD, 2015). 


\section{Nativi digitali tra mito e realtà}

La Svizzera è oggi tra gli stati con maggiore espansione digitale, sia in termini di connettività che di dispositivi di dispositivi personali: già nel 2012 la quasi totalità della popolazione dichiara di avere accesso ad almeno un computer in casa (Organisation for Economic Co-operation and Development [OECD], 2015); se prima dei 9 anni il possesso di uno smartphone riguarda già il $98 \%$ della popolazione (Suter et al., 2015), questa cifra nei giovani tra i 12 e i 19 anni è il $100 \%$, con pochissime variazioni dovute allo stato socio-economico o ad altri fattori (Waller, Willemse, Genner, Suter \& Süss, 2016); alcuni studi sembrano anzi mostrare che l'uso della rete sia più intenso per giovani con origine sociale meno avvantaggiata (Camerini, Schulz \& Jeannet, 2017). Una metà dei nostri giovani già a partire da 9-10 anni usa regolarmente dispositivi digitali e internet, strumenti che diventano semplicemente scontati e sempre presenti a partire dai 15 anni (OECD, 2015). Possiamo considerare tale ampia diffusione dell'accesso alle tecnologie quale garanzia sufficiente per lo sviluppo di solide competenze digitali nei giovani?

All'inizio degli anni 2000 si è infatti diffuso il concetto di nativo digitale (Prensky, 2001a; 2001b; Tapscott, 1998), per descrivere i giovani della nuova generazione, che avrebbero mutato i propri comportamenti e processi di apprendimento in funzione della disponibilità di internet e del web. La metafora proposta è linguistica: un parlante nativo non presenta alcun accento o inflessione, e usa la lingua con assoluta fluidità e naturalezza; così avverrebbe per chi è «nato con i media digitali» ed è quindi un nativo digitale. Al contrario, un immigrato, anche se ben inserito e con buone competenze linguistiche, manterrà nella maggior parte dei casi alcuni tratti linguistici non standard che lo identificheranno subito come tale; analogamente, così avverrebbe per chi appartiene a generazioni precedenti: i cosiddetti «immigrati digitali». Le scuole avrebbero dunque accolto una generazione di allievi naturalmente più competenti dei propri docenti, capaci, se posti in condizioni adeguate, di sfruttare al meglio e in autonomia l'enorme potenziale delle tecnologie digitali.

Una parte del mondo accademico ha accolto questa classificazione, e si è impegnata nel definire quali fossero le caratteristiche di un nativo digitale, arrivando ad esempio a sviluppare e validare la Digital Natives Assessment Scale (Teo, 2013), applicata poi in diversi studi (ad es. Teo, 2016; Yong \& Gates, 2014). Secondo questi autori, la generazione di appartenenza è rilevante per le competenze digitali di un individuo, e questo implica la necessità di un ripensamento delle modalità di insegnamento e di apprendimento a tutti i livelli, a partire dalla scuola dell'obbligo (Palfrey \& Gasser, 2008). Altri hanno invece sollevato voci critiche, che hanno cercato di demistificare l'idea di salto evolutivo, mostrando come la cosiddetta net generation non possieda in realtà di per sé competenze digitali avanzate (cf. Bullen, Morgan, Belfer \& Qayyum, 2009; Bullen, Morgan \& Qayyum, 2011; Schulmeister, 2008; Thompson, 2013). Alcuni autori hanno messo in evidenza come l'appartenenza generazionale non 
sia né l'unico, né il più importante indicatore di competenza digitale: Helsper \& Eynon (2009) dimostrano che altri fattori quali genere, ampiezza d'uso delle tecnologie e livello di educazione sono cruciali, se dobbiamo definire chi è «nativo digitale» nel senso di chi dimostra competenze digitali solide e approfondite. $\mathrm{E}$ da considerare inoltre una disconnessione tra l'uso delle tecnologie nella vita privata (in chiave ludica) e a scuola o nella professione (Selwyn, 2006; Wang, Hsu, Campbell, Coster \& Longhurst, 2014). La scuola non deve dunque tanto adattarsi alle inclinazioni di una generazione diversa dalle precedenti, ma accettare la sfida di educare in maniera coerente ed efficace a un uso sano e adeguato degli strumenti digitali.

Nel 2010, Chris Jones ha curato un numero tematico di Learning, Media and Technology che ha provato a costruire una panoramica integrata sulle competenze digitali dei giovani, e ha concluso che

(...) young people at school and university use technologies in ways that are related to their purposes and exhibit a diversity that contrasts with the idea of a sharp generational change. (...) there are significant age-related changes but (...) these changes are mediated by the active appropriation of technology by young people who act purposively and in relation to influential institutional contexts. (Jones, 2010, p. 368)

L'ampio studio etnografico di Livingstone e Sefton-Green (2016) conferma questa ipotesi, documentando, all'interno di una stessa classe di scuola secondaria, modalità molto diverse di appropriazione delle tecnologie, e di integrazione di pratiche digitali nei contesti sociali e familiari. Un recente studio (Botturi, sous presse) mostra analogamente che classi diverse, anche nel medesimo istituto e composte da allievi di simile provenienza socio-economica, presentano profili digitali molto diversi. Nonostante significativi episodi di sintesi, il dibattito resta aperto, in uno scenario di continuo mutamento tecnologico e di rapida evoluzione delle pratiche di socializzazione degli strumenti e dei contenuti digitali.

Quella che potrebbe sembrare una querelle accademica, ha in realtà importanti riflessi nella pratica educativa quotidiana nelle scuole. Da un lato, infatti, è esperienza comune tra i docenti la scoperta di una scarsa competenza digitale dei propri allievi anche in attività semplici, dalla scrittura di un'email (che viene interpretata come un lungo "messaggino») alla stesura di un curriculum, dalla selezione di informazioni in rete all'uso di un foglio di calcolo. D'altro canto, quando si trovano sul delicato terreno delle tecnologie digitali, molto spesso i docenti provano riverenza e quasi timore nel confronto con i loro allievi, ritenuti comunque "più avanti» e più aggiornati.

\section{Competenze digitali?}

Un punto spesso trascurato nel dibattito sui nativi digitali è la definizione stessa di competenza digitale. Se gli studi accademici si concentrano spesso su un aspetto puntuale (l'uso di Twitter, o le competenze di ricerca di informazioni 
in rete, ecc.), l'ampiezza e la pervasività dell'uso delle tecnologie digitali oggi hanno portato però a distinguere e articolare il contenuto di termini come digital literacy, information literacy, media literacy, technology literacy e digital competences (cf. Hobbs, 2010). Se da un lato troviamo sicuramente le conoscenze di base sul funzionamento e l'uso dei computer e della rete (technology literacy), dall'altro troviamo anche l'uso dei media "consapevolmente e in maniera responsabile», che "comprende il saper soddisfare i propri bisogni di informazione e svago attraverso i media, ma anche l'essere in grado di giudicare con criterio sia i media che il consumo che se ne fa" (Giovani e Media, 2016, p. 6). In mezzo si collocano le competenze di ricerca e uso di informazione (information literacy), che vanno però lette anche in chiave di produzione e diffusione di testi multimediali.

Dal punto di vista scolastico, si tratta dunque sia di competenze tecniche, che afferiscono in maniera più o meno definita al campo dell'informatica, ma anche di competenze creative e relazionali, legate ai temi della salute e della cittadinanza. Questa trasversalità si ritrova anche nei nuovi piani di studio HarmoS delle tre regioni linguistiche (Plan d'études romand; Lehrplan21 e Piano di studio della scuola dell'obbligo): i tre documenti identificano l'area delle competenze digitali come un settore trasversale alle discipline, per poi definire soluzioni diverse di integrazione operativa di attività per lo sviluppo effettivo delle competenze digitali nell'organizzazione scolastica.

\section{Lo studio ICILS}

L'indagine ICILS (International Computer and Information Literacy Study) ha come obiettivo valutare l'alfabetizzazione informatica e comunicazionale dei giovani adolescenti al loro ottavo anno di scolarizzazione; in Ticino, la terza media (Calvo \& Zampieri, 2017, p. 6). A livello globale questa indagine ha coinvolto complessivamente 20 Stati, circa 60'000 studenti e 20'000 tra docenti, direttori e responsabili informatici di sede (Fraillon, Ainley, Schulz, Friedman \& Gebhardt, 2014, p.33).

Gli obiettivi dell'indagine sono molteplici: in primo luogo poter effettuare dei confronti tra i paesi (come anche all'interno dei paesi) riguardo le competenze raggiunte dagli allievi. In seguito verificare come gli aspetti istituzionali, infrastrutturali o relazionali in campo di educazione, contribuiscano allo sviluppo delle conoscenze informatiche. Un altro obiettivo è quello di individuare quali, tra le conoscenze informatiche in possesso degli allievi, sono frutto di insegnamenti scolastici e quali altre, invece, sono state acquisite tramite fonti di informazione alternative. Infine, vi è anche l'individuazione di possibili legami tra il livello di competenza raggiunto dagli allievi e determinati fattori contestuali.

L'importanza attribuita ai fattori contestuali è dovuta alla consapevolezza che le competenze informatiche e comunicazionali sono acquisite, ancor più di quelle connesse con altre discipline, attraverso una grande varietà di attività e di 
esperienze che avvengono nelle situazioni sociali più disparate (Ainley, Enger \& Searle, 2008). Per questo nell'indagine sono distinti i seguenti livelli: (Fraillon et al., 2014).

1. Individuale: che concerne le caratteristiche proprie dello studente e del suo processo di apprendimento.

2. Familiare: che riguarda le peculiarità economiche, sociali e culturali della famiglia dell'allievo.

3. Scolastico: che concerne le infrastrutture delle sedi scolastiche, così come i metodi insegnamento in atto.

4. Collettivo/nazionale: che concerne le caratteristiche economiche, sociali ed educative della collettività di riferimento dell'allievo.

Questi livelli contestuali hanno a loro volta elementi statici e dinamici.

1. Gli antecedenti: i fattori esogeni, sostanzialmente immutabili, che non possono essere influenzati dal processo di apprendimento dell'allievo. Esempi tipici sono il contesto tecnologico del Paese, la dotazione infrastrutturale della sede scolastica o lo statuto socio-economico dell'allievo.

2. I processi: sono i fattori che influenzano direttamente il processo di apprendimento dell'allievo e che a loro volta sono condizionati dagli antecedenti. Esempi tipici sono i metodi di insegnamento e l'uso delle Tecnologie dell'Informazione e della Comunicazione (TIC) da parte degli allievi.

\begin{tabular}{|c|c|c|}
\hline ANTECEDENTI & PROCESSI & RISULTATI \\
\hline $\begin{array}{l}\text { Collettivi/nazionali } \\
\text { Sistema educativo, } \\
\text { Infrastruttura economica e } \\
\text { tecnologica nazionale. } \\
\text { Scolastici }\end{array}$ & $\begin{array}{l}\text { Collettivi/nazionali } \\
\text { Politiche educative inerenti } \\
\text { le TIC. } \\
\text { Scolastici }\end{array}$ & \\
\hline $\begin{array}{l}\text { Furriculum formativo. } \\
\text { Familiari }\end{array}$ & Familiari & $\begin{array}{l}\text { Alfabetizzazione } \\
\text { informatica e } \\
\text { comunicazionale }\end{array}$ \\
\hline $\begin{array}{l}\text { Statuto socio-economico } \\
\text { della famiglia e disponibilità } \\
\text { di infrastrutture TIC a casa. }\end{array}$ & Uso delle TIC a scuola & \\
\hline $\begin{array}{l}\text { Individuali } \\
\text { Caratteristiche dell'allievo. }\end{array}$ & $\begin{array}{l}\text { Individuali } \\
\text { Processo di apprendimento }\end{array}$ & \\
\hline
\end{tabular}

Figura 1: Fattori contestuali in ICILS

Lo schema evidenzia come, a differenza degli antecedenti, i processi possano subire delle retroazioni dall'acquisizione di specifiche competenze di alfabetizzazione informatica e comunicazionale: queste ultime, infatti, possono portare dei mutamenti negli stili di consumo delle TIC da parte degli allievi.

Nell'indagine ICILS la Computer and Information Literacy (CIL) è definita come l'insieme delle competenze che un individuo deve acquisire per rispondere 
ai requisiti della società della comunicazione (Eickelmann, Bos, Gerick \& Kahnert, 2014; Erstad, 2004). Non è questo il luogo per approfondire tale costrutto, ma sarà sufficiente notare che questa definizione operazionalizza e sintetizza un lungo percorso di definizione delle nuove literacy legate al mondo digitale, e che si colloca all'interno di diversi modelli attualmente discussi in questo ambito (Senkbeil et al., 2014).

In ICILS si fa quindi riferimento a un modello che prevede due grandi aree, che entrambe comprendono competenze tecniche e conoscenze intellettuali: (a) la raccolta e la gestione di informazioni e (b) la loro produzione e condivisione (Audunson \& Nordlie, 2003; Fraillon, Schulz \& Ainley, 2013). Questo concetto di alfabetizzazione informatica e comunicazionale (oggi diremmo piuttosto di digital literacy) è stato conseguente allo sviluppo, dalla seconda metà degli anni 2000, del cosiddetto Web 2.0 (O’Reilly, 2005) che ha portato, con il proliferare dei social network e le piattaforme di condivisione di media, alla trasformazione degli utenti di internet da fruitori passivi di contenuti a soggetti attivi, capaci di produrre e diffondere conoscenza. Concretamente, il modello prevede l'articolazione di due macro-dimensioni: la raccolta e la gestione delle informazioni e la loro successiva trasformazione e condivisione.

Questo studio, appoggiandosi ai dati rilevati da ICILS in Ticino, cerca di rispondere alle seguenti domande: Quali caratteristiche del sistema educativo favoriscono lo sviluppo di competenze digitali? Esiste una relazione tra età di inizio dell'uso delle tecnologie, frequenza d'uso e tipologia d'uso e le competenze digitali nei diversi ambiti?

\section{Le tecnologie digitali nella scuola ticinese}

Questo studio approfondisce alcuni aspetti relativi allo sviluppo delle competenze digitali nei giovani a partire dai dati rilevati nello studio ICILS. In particolare, si concentra sui dati ticinesi interrogandosi su quali competenze digitali vengono sviluppate fuori dal contesto scolastico e su quali fattori influiscano sul loro sviluppo. Per comprendere le analisi, i risultati e le implicazioni qui presentate è bene dunque tratteggiare la situazione della scuola ticinese al tempo dell'indagine ICILS 2013 (Eickelmann et al., 2014).

\section{Fattori legati al sistema educativo}

Un dato strutturale relativo ai fattori contestuali di tipo collettivo-nazionale è sicuramente l'inserimento di materie legate alle TIC all'interno dei curricoli scolastici obbligatori. La Tabella 1 presenta per gli stati partecipanti a ICILS (a) il livello di centralizzazione nazionale/regionale rispetto alle decisioni inerenti l'integrazione delle TIC nell'insegnamento (nazionale/regionale/nessuna integrazione), (b) la presenza o meno di una materia legata all'informatica e/o alla comunicazione e il suo carattere opzionale o obbligatorio nella scuola 
primaria (ISCED 1) e nel secondario I (ISCED 2), e (c) la presenza di una valutazione formale delle competenze informatiche e comunicazionale all'ottavo anno di scolarità. I dati della tabella offrono unicamente una visione parziale, dal momento che le informazioni raccolte sono forzatamente superficiali (non considerano, ad esempio, le differenze regionali) e non riescono a dare una dimensione qualitativa e quantitativa dell'importanza di queste discipline nei diversi curricula nazionali. Consideriamo però i dati relativi a Repubblica Ceca, Australia e Polonia, i tre Stati che hanno ottenuto i risultati migliori nei punteggi ICILS: questi risultati sembrano suggerire l'esistenza di politiche nazionali volte ad inserire nei curriculi formativi delle discipline legate alle TIC, obbligatorie e valutate formalmente, possa favorire un buon esito del test ICILS.

Tabella 1: Elaborazione: Fraillon et.al, 2014/Calvo \& Zampieri, 2017

\begin{tabular}{llllcl} 
& & & & \multicolumn{3}{c}{$\begin{array}{c}\text { Valuta- } \\
\text { zione } \\
\text { TIC 8 }\end{array}$} \\
& Liv. decisionale TIC & ISCED 1 & ISCED 2 & anno & Denominazione \\
\hline Repubblica Ceca & Nazionale e regionale & Obbligatoria & Obbligatoria & Sì & Informatica \\
Australia & Nazionale e regionale & Obbligatoria & Obbligatoria & Sì & Varie opzioni \\
Polonia & Nazionale e regionale & Obbligatoria & Obbligatoria & Sì & Scienze informatiche \\
Norvegia & Nazionale e regionale & Inesistente & Inesistente & No & Inesistente \\
Corea del Sud & Assente & Inesistente & Opzionale & No & Informatica \\
Germania & Nazionale e regionale & Inesistente & Opzionale & No & Informatica \\
Slovacchia & Nazionale e regionale & Obbligatoria & Obbligatoria & Sì & Informatica \\
Russia & Nazionale e regionale & Inesistente & Obbligatoria & No & Informatica e TIC \\
Croazia & Nazionale e regionale & Inesistente & Obbligatoria & Sì & Scienze informatiche \\
Slovenia & Solo nazionale & Inesistente & Opzionale & Sì & Studi informatici \\
Ticino & Solo regionale & Inesistente & Opzionale & No & Varie opzioni \\
Lituania & Nazionale e regionale & Inesistente & Obbligatoria & Sì & tecnologie dell'info. \\
Cile & Nazionale e regionale & Obbligatoria & Obbligatoria & Sì & Educ. Tecnologica \\
Tailandia & Solo nazionale & Obbligatoria & Obbligatoria & Sì & TIC \\
Turchia & Nazionale e regionale & Obbligatoria & Obbligatoria & Sì & Tecnologie \\
& & & & & \\
\hline & & & & &
\end{tabular}

In Ticino dunque il livello decisionale è quasi unicamente regionale (se escludiamo le linee guida generali tracciate da HarmoS) e le materie legate alle TIC sono assenti alle scuole elementari. Per la scuola media (livello secondario I) non esiste, di fatto, una materia esplicitamente consacrata all'insegnamento delle TIC. L'acquisizione di queste competenze è demandato alle singole materie, anche se occorre segnalare l'esistenza di un corso di alfabetizzazione informatica 
(12 unità didattiche) in prima media, che però non è valutato e ha una dotazione oraria esigua. Elementi riferibili alle nuove tecnologie sono riscontrabili solo in alcune opzionali come "attività tecniche» e, soprattutto, "attività commerciali». Non è, inoltre, prevista una valutazione specifica sulle competenze informatiche e comunicazionali degli allievi in terza media ( $8^{\circ}$ anno).

\section{Evoluzione della politica educativa}

In questo paragrafo saranno contestualizzati meglio alcuni aspetti emersi dall'indagine con l'evoluzione della politica educativa in questo ambito. È bene notare fin da subito che l'indagine ICILS è stata condotta proprio all'inizio di un periodo di transizione del sistema scolastico cantonale, che negli anni successivi si è trovato confrontato con l'introduzione del nuovo Piano della scuola dell'obbligo Ticinese (Dipartimento dell'educazione, della cultura e dello sport [DECS], 2015a), e con la proposta di riforma a livello sistemico contenuta nel documento La scuola che verrà (DECS, 2015b).

Innanzitutto ci sembra interessante riflettere sulle corrispondenze tra il modello teorico alla base di ICILS 2013 e (a) il Piano di formazione della Scuola Media del 2004 (PF2004) (DECS, 2004) in vigore durante l'amministrazione dell'inchiesta e (b) il Piano della scuola dell'obbligo (PSO2015) presentato nel 2015 (Tabella 2).

\section{Tabella 2: Competenze digitali in ICIL e nel piano di formazione}

\begin{tabular}{|c|c|c|}
\hline & Modello ICILS 2013 & Piano di formazione 2004 \\
\hline Sapere & $\begin{array}{l}\text { Reperire e selezionare le } \\
\text { informazioni. }\end{array}$ & $\begin{array}{l}\text { - Integrazione dell'informatica nelle discipline } \\
\text { - Alfabetizzazione informatica ( } 12 \text { ore) }\end{array}$ \\
\hline Saper fare & $\begin{array}{l}\text { Elaborare e trasformare le } \\
\text { informazioni. }\end{array}$ & $\begin{array}{l}\text { - Integrazione dell'informatica nelle discipline } \\
\text { - Alfabetizzazione informatica ( } 12 \text { ore) }\end{array}$ \\
\hline Saper essere & Condividere le informazioni & - Attività commerciali (opzione) \\
\hline
\end{tabular}

Il PF2004, attraverso la Riforma 3, prevede un corso di alfabetizzazione informatica di 12 ore in prima media incentrato sugli «aspetti funzionali di base relativi all'uso del PC»; sull'«educazione all'organizzazione dei materiali informatici» e su «aspetti etici: uso di Internet». Esso demanda, inoltre, ai docenti delle diverse discipline integrare l'informatica al fine di «... educare le giovani generazioni ad un uso consapevole delle tecnologie e alla capacità di selezionare ed utilizzare il sapere e l'informazione». Infine propone l'attività opzionale "Attività commerciali», nella quale l'informatica è integrata in maniera strutturale e con un profilo definito abbastanza definito di competenze.

Il PSO2015, che è praticamente coevo all'indagine ICILS 2013, concettualizza le TIC come degli strumenti che non solo permettono alle persone di entrare in contatto con una grande quantità di informazioni, ma anche di proporne di nuove e di scambiarle attraverso canali che consentono oramai a 
chiunque di superare la semplice comunicazione punto a punto. Da un punto di vista pratico tuttavia la formazione alle TIC resta, almeno a livello di terzo ciclo, ancorata agli spazi trasversali alle discipline, in maniera sostanzialmente analoga a quanto avviene con il PF2004. Un'importante novità è costituita anche grazie all'entrata in vigore dell'accordo HarmoS - dalla definizione di attività volte a integrare le TIC anche nell'insegnamento primario.

\section{Dotazione tecnologica delle scuole}

Dal punto di vista della disponibilità di tecnologie digitali nelle sedi scolastiche, la scuola ticinese si presenta diversificata. Ancora oggi, sono poche le sedi di scuola elementare che dispongono di un'aula informatica e ancor meno quelle con un collegamento internet. Le sedi di scuola media hanno una dotazione informatica generalmente migliore, ma solo a partire dal 2015-16 il Cantone ha deciso di investire per iniziare a dotare tutte le sedi scolastiche di collegamento internet e di rete WiFi. Recentemente il DECS, recependo le indicazioni del rapporto e-education (Gruppo di lavoro e-education, 2012) ha costituito un centro di competenza che ha tra i suoi obiettivi di rendere il comparto tecnologico delle scuole più adeguato e conforme a delle finalità educative.

Per quanto riguarda la dotazione infrastrutturale TIC delle scuole medie ticinesi, possiamo dire che essa appare sostanzialmente simile a quella della maggior parte dei paesi europei coinvolti nell'indagine, ma probabilmente inferiore a quella di Stati con una ricchezza pro-capite e un indice di sviluppo tecnologico simili alla Svizzera, ad esempio Germania, o Norvegia.

\section{ICILS in Ticino}

I dati raccolti nello studio ICILS in Ticino descrivono dunque una situazione particolare: gli indicatori rilevati per la computer e information literacy si riferiscono in questo caso a competenze sviluppate dai giovani sostanzialmente fuori dalla scuola (che non se ne occupa se non raramente), in contesti di educazione informale, autonomamente o in relazione con dei pari. I risultati che emergono dunque sono solo in minima parte ascrivibili a un'esplicita azione educativa della scuola.

Se prendiamo come valida l'ipotesi dell'esistenza di un salto generazionale e di nativi digitali per definizione competenti, potremmo aspettarci un buon livello di competenze digitali. Se invece crediamo nell'importanza di un percorso di educazione formale per lo sviluppo di competenze digitali solide, ci aspetteremo un risultato piuttosto scarso.

La legge scolastica ticinese assegnata alla scuola assolve un ruolo di eguaglianza educativa, che le chiede di portare ogni allievo ad almeno un livello minimo di competenza. La scuola ticinese si assume consapevolmente questo compito e si è configurata come agenzia educativa che tenta di eliminare le diseguaglianze sociali dovute al livello socio-economico o alle condizioni familiari e di vita dei giovani. Questo è confermato anche dai risultati PISA 2012, dove si può 
vedere che la dispersione dei punteggi ottenuti dagli allievi è tra le più basse, soprattutto in matematica. In breve, ciò significa che le medie dei singoli allievi si concentrano di più attorno alla media del Canton Ticino mentre in altri paesi i risultati si disperdono di più (Salvisberg \& Zampieri, 2015). Tuttavia, la situazione esposta sopra, cioè la sostanziale non copertura delle competenze digitali nell'ambito dei programmi e delle pratiche di insegnamento nelle scuole primarie e secondarie, suggerisce che l'effetto di questo ruolo di eguaglianza sociale non sarà visibile nell'ambito dello sviluppo di competenze digitali. Nelle sezioni successive terremo conto di questo elemento interpretativo nell'analisi dei risultati dello studio ICILS per il Ticino.

\section{Metodologia}

\section{Campione del Canton Ticino}

A livello svizzero era inizialmente previsto che partecipassero all'inchiesta circa 3'000 allievi e 1'000 professionisti della scuola. La comparazione sarebbe dovuta avvenire tra le tre regioni linguistiche. La somministrazione non è però potuta avvenire correttamente principalmente per la mancanza di un supporto politico nazionale, che ha indotto interi cantoni e numerose sedi a rinunciare alla loro partecipazione. Alcune ragioni che possono aver indotto le istituzioni federali a non fornire un adeguato sostegno allo studio sono ben sintetizzate da Bottani (2015).

Il Canton Ticino rappresenta da solo la regione linguistica italofona della Svizzera, ragion per cui è stato oggetto di una sovra-campionatura, eseguita secondo i parametri previsti dall'indagine ICILS. Complessivamente hanno preso parte all'indagine circa 1'000 allievi e 300 tra docenti, direttori e responsabili informatici provenienti da 20 sedi. Gli istituti scolastici sono stati selezionati sulla base di criteri legati alla loro dimensione e al loro posizionamento geografico in modo da essere rappresentativi della realtà regionale; per ogni sede sono stati sorteggiati (laddove le dimensioni lo consentivano) 60 allievi e 15 docenti. Per ciò che concerne gli studenti, è stato fatto in modo che il genere e l'anno di nascita fossero ripartiti in modo da essere rappresentativi della sede, mentre per ciò che concerne i docenti, oltre a ciò, è stata considerata anche la materia insegnata. Questa scelta di campionatura rende statisticamente legittimo e scientificamente valido il confronto dei risultati ticinesi con i risultati degli altri paesi coinvolti nello studio (Calvo \& Zampieri, 2017).

\section{Strumenti di raccolta dati}

La raccolta dati del progetto ICILS è stata affidata a due strumenti: i questionari e un test modulare sulle competenze informatiche e comunicazionali (Eickelmann et al., 2014). Come anticipato, i fattori di contesto sono rilevati principalmente attraverso i questionari. L'indagine ne propone quattro (Fraillon et al., 2014). 
1. Uno rivolto agli studenti che verte sulle caratteristiche familiari, sull'esperienza nell'uso delle TIC a casa e a scuola e sulle loro attitudini a riguardo;

2. uno proposto agli insegnanti, incentrato sull'autovalutazione delle loro competenze nell'uso delle TIC, sull'uso che ne fanno a scuola, sulle loro attitudini a riguardo e sulle infrastrutture messe loro a disposizione;

3. uno destinato ai responsabili informatici di sede, focalizzato sulle risorse TIC disponibili in sede e sul supporto tecnico e pedagogico a disposizione dei docenti in questo ambito;

4. uno rivolto ai direttori di sede che indaga sulle strategie di integrazione delle TIC nell'insegnamento.

Le finalità del presente articolo ci portano a considerare unicamente le dimensioni di processo - in particolare per quanto riguarda l'uso scolastico e domestico delle TIC - rilevabili a partire dal questionario rivolto agli studenti.

Probabilmente l'aspetto più innovativo dell'indagine ICILS 2013 è costituito dalle attività che gli allievi sono chiamati a svolgere e che costituiscono lo strumento per definire e misurare, in una situazione di realtà simulata, le loro competenze informatiche e comunicazionali (Fraillon et.al, 2014). Il test consiste in quattro moduli, ciascuno dei quali della durata di 30 minuti; ogni studente è chiamato a svolgerne due. Ogni modulo è costituito da un insieme coerente di domande e proposte di attività basate su un tema legato ad un aspetto della vita reale e strutturato secondo un processo narrativo lineare. Ciascun modulo inizia con una serie di piccoli esercizi (lo svolgimento dei quali richiede mediamente un minuto) e termina con un'attività più estesa della durata di 15-20 minuti. Le attività sono: l'organizzazione di attività extra-scolastiche, l'organizzazione di un concorso di band musicali, una presentazione sul funzionamento della respirazione umana e infine la pianificazione di una gita scolastica. Gli esercizi implicano competenze tecniche, recettive, produttive, valutative e sottendono ad un uso sicuro ed etico delle informazioni. Complessivamente ogni modulo attribuisce un massimo di 62 punti.

Per ciò che concerne le due grandi aree di competenza cui si è fatto riferimento in precedenza, gli esercizi sono ripartiti come nella Tabella 3.

\section{Tabella 3: Aree di competenza nei moduli del test ICILS}

Raccolta e gestione delle informazioni: 33\% Produzione e scambio di informazioni: 67\%

Conoscenze e usi dell'infrastruttura informatica: $13 \%$

Accedere a delle informazioni e valutarle: $15 \%$

Gestire le informazioni: 5\%
Trasformare le informazioni: $17 \%$

Creare informazioni: $37 \%$

Condividere informazioni in modo sicuro: $13 \%$ 


\section{Risultati}

Le analisi proposte in questo articolo considerano i dati raccolti tramite il questionario, letti in relazione ai punteggi complessivi ottenuti dai partecipanti nel test. Si è scelto di proporre analisi descrittive perché più pertinenti per affrontare le domande di ricerca individuate. Dopo un primo confronto con i dati internazionali, si è dunque trattato di approfondire innanzi tutto la relazione tra le competenze e l'età in cui gli allievi dichiarano di aver iniziato a utilizzare strumenti tecnologici, e la tipologia o finalità d'uso.

\section{Confronto internazionale}

Il confronto internazionale di ICILS mette in relazione i risultati medi ticinesi con quelli dei quattordici Stati che hanno adempiuto ai criteri di campionatura (Calvo \& Zampieri, 2017). I dati sono presentati nella Tabella 4. La colonna a sinistra indica i punteggi medi ponderati ottenuti nei diversi Stati, quella a destra l'errore standard. I punteggi segnati in bianco rientrano nell'intervallo del livello di competenza 1, quelli in grigio in quello del livello di competenza 2, mentre quelli in grigio scuro hanno un livello di competenza inferiore.

Tabella 4: Confronto internazionale. Elaborazione: Fraillon et.al, 2014/CIRSE, 2015

\begin{tabular}{lcl} 
Paese & Punteggio medio & e.s \\
\hline Repubblica Ceca* $^{*}$ & 553 & 2.1 \\
Australia* & 542 & 2.3 \\
Polonia* $^{*}$ & 537 & 2.4 \\
Norvegia $^{1 *}$ & 537 & 2.4 \\
Corea del Sud* $^{*}$ & 536 & 2.7 \\
Germania* $^{*}$ & 523 & 2.4 \\
Slovacchia* $^{*}$ & 517 & 4.6 \\
Russia* $^{*}$ & 516 & 2.8 \\
Croazia* & 512 & 2.9 \\
Slovenia* & 511 & 2.2 \\
Ticino & 496 & 4.3 \\
Lituania & 494 & 3.6 \\
Cile \# & 487 & 3.1 \\
Tailandia \# & 373 & 4.7 \\
Turchia \# & 361 & 5.0 \\
\hline
\end{tabular}


Gli Stati segnati con il simbolo asterisco $\left(^{*}\right)$ fanno registrare un punteggio medio superiore al Ticino statisticamente significativo; quelli con il segno cancelletto (\#) un punteggio medio inferiore statisticamente significativo $(\mathrm{p}<0.5)$. Complessivamente, la comparazione dei punteggi medi evidenzia come - escludendo la Tailandia e la Turchia, notevolmente staccate - la maggioranza degli Stati ha risultati relativamente omogenei.

Il Ticino, con 496 punti, non si discosta in maniera statisticamente significativa dalla media internazionale, inoltre, assieme a 11 sistemi educativi su 14 ha ottenuto un punteggio medio che si inserisce nell'intervallo di competenza di Livello 2. Occorre però notare che la media raggiunta si avvicina molto al limite inferiore di questo livello, che è di 492 punti, mentre nei cinque Stati ove si sono registrati i risultati migliori - Repubblica Ceca, Australia, Polonia, Norvegia e Corea del Sud - i punteggi medi si avvicinano maggiormente al limite con il livello superiore, che è 576 punti.

Secondo le definizioni di ICILS (Fraillon et al., 2014), un allievo con un livello di competenza 2 è in grado di utilizzare i più comuni applicativi informatici, è consapevole dell'esistenza di rischi a proporre informazioni personali sul web, ma non ha piena capacità di proteggersi in questo ambito. $\mathrm{E}$ in grado di ricercare informazioni su internet e ha coscienza che non sempre sono affidabili, ma non possiede gli strumenti per selezionarle correttamente. Può realizzare autonomamente semplici attività che richiedono l'uso delle TIC, ma ha bisogno di supporto per svolgerne di più complesse. Non ha, infine, praticamente nessuna consapevolezza di aspetti legali, come quelli legati alla proprietà intellettuale o alla privacy.

Il raffronto della distribuzione interna dei punteggi all'interno dei diversi sistemi educativi mostra una situazione abbastanza differenziata e non corrispondente alla graduatoria internazionale. La Figura 2 illustra la deviazione standard dei punteggi, che è indicatrice della distribuzione dei punteggi all'interno dei singoli Stati. Il grafico presenta, inoltre, la ripartizione nei diversi percentili della popolazione. 


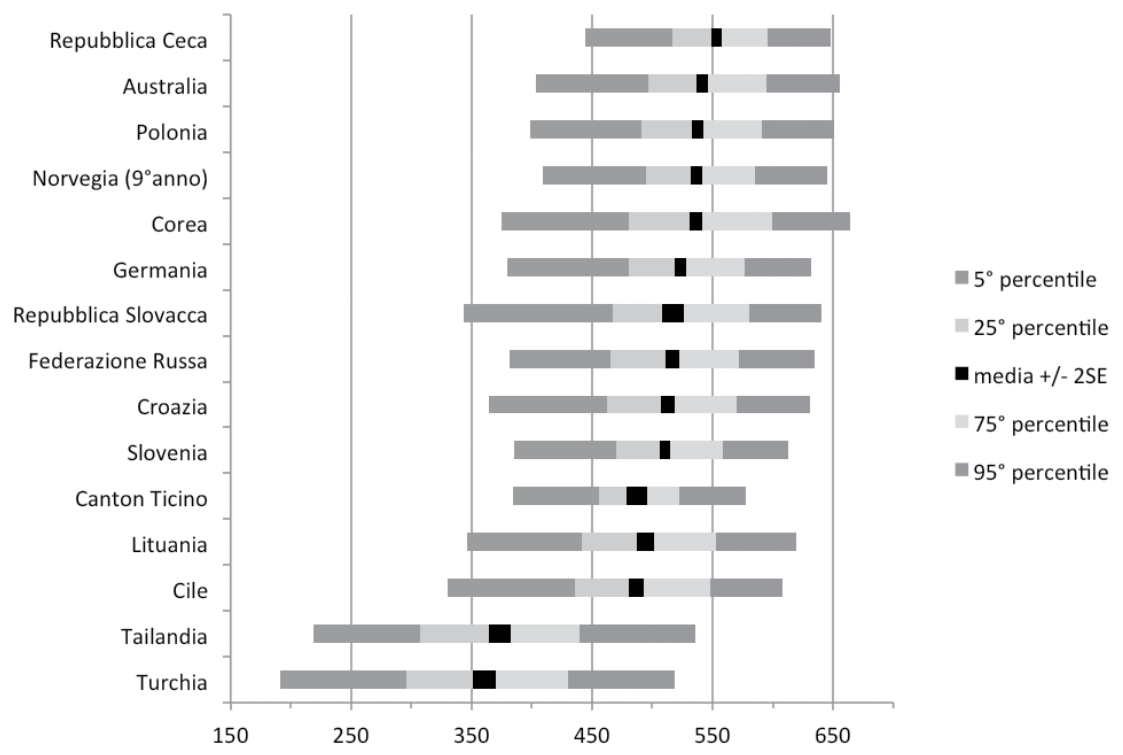

Figura 2: Distribuzione dei punteggi. Elaborazione: Fraillon et.al, 2014/CIRSE, 2015.

Il Ticino, con 62 punti di deviazione standard al pari della Repubblica Ceca, fa registrare la minore differenziazione interna dei punteggi. Questo corrisponde, tra l'altro, alla minor differenziazione interna rilevata per i punteggi svizzeri nel confronto internazionale, sia in base al genere (Lorenz, Gerick, SchulzZander \& Eickelmann, 2014), sia in base all'origine socio-economica (Wendt, Vennemann, Schwippert \& Drossel, 2014).

Un altro indicatore conseguente è dato dal raffronto tra i risultati medi ottenuti dal 5\% degli allievi che ha ottenuto i punteggi peggiori e dal $5 \%$ che ha ottenuto quelli migliori: lo scarto per il Ticino è infatti di 110 punti, mentre quello medio di ICILS 2013 è di 258 punti.

È difficile interpretare questo risultato in termini comparativi perché - come vedremo anche nei paragrafi consacrati ai fattori contestuali di tipo collettivo/ nazionale e familiare - il Ticino è una regione demograficamente limitata e istituzionalmente, socialmente ed economicamente molto più omogena rispetto agli Stati che partecipano all'indagine ICILS 2013.

La ripartizione degli allievi tra i diversi livelli di competenza mostra che il Ticino ha una percentuale relativamente alta rispetto agli altri partecipanti: l'82\%, di allievi che si situano entro il Livello 1 e il Livello 2 di competenza. In Ticino, quindi, troviamo solo il $9 \%$ degli studenti con competenze informatiche e comunicazionali inferiori al Livello 1, cioè veramente scarse (Figura 3). Una percentuale simile si registra anche in Corea del Sud, che ha però un risultato 
medio sensibilmente superiore. Non imprevedibilmente, si può anche notare una proporzione abbastanza scarsa di allievi molto competenti, che raggiungono almeno il Livello 3, anche in questo caso solo il 9\%, un dato inferiore, ad esempio, a quello della Lituania e del Cile che hanno risultati medi complessivi inferiori o analoghi. In particolare, nessun allievo ticinese ha raggiunto il Livello 4.

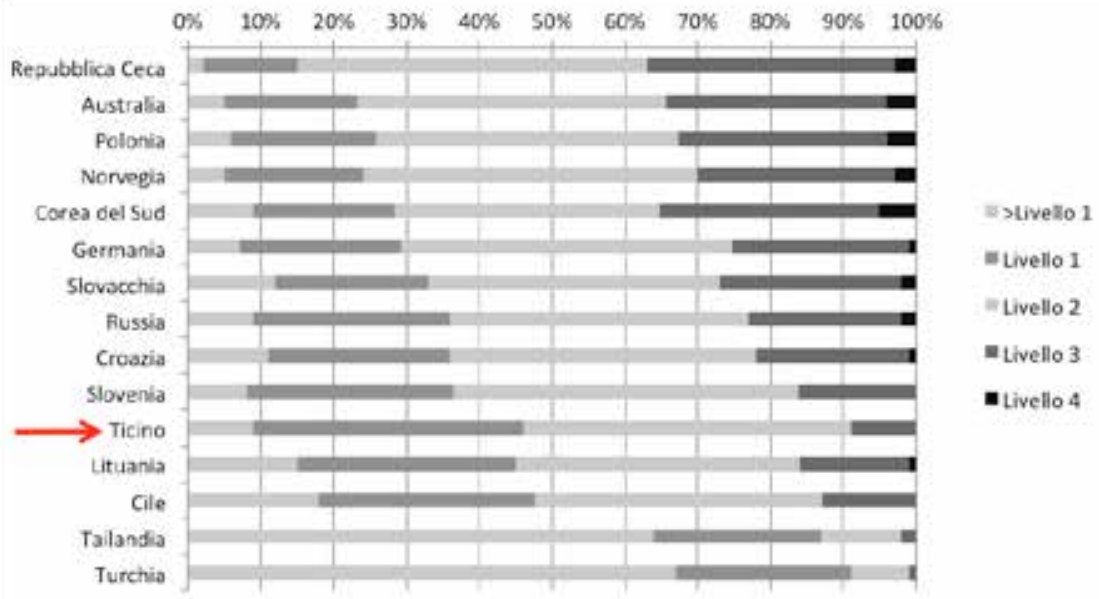

Figura 3: Distribuzione per livelli di competenza. Elaborazione: Fraillon et.al, 2014/CIRSE, 2015.

Dopo averli collocati nel contesto internazionale, in questi paragrafi cercheremo di analizzare i dati ticinesi segmentando il campione secondo diverse dimensioni, per identificare eventuali correlazioni.

\section{Età di primo uso}

Il primo aspetto preso in considerazione è l'età d'uso: potremmo aspettarci che, quanto prima un giovane inizia a usare le tecnologie, tanto più avrà competenze digitali solide una volta raggiunti i 13 anni. La Figura 3 riporta i punteggi ottenuti dagli allievi in relazione all'età autodichiarata di inizio di uso delle tecnologie digitali. 


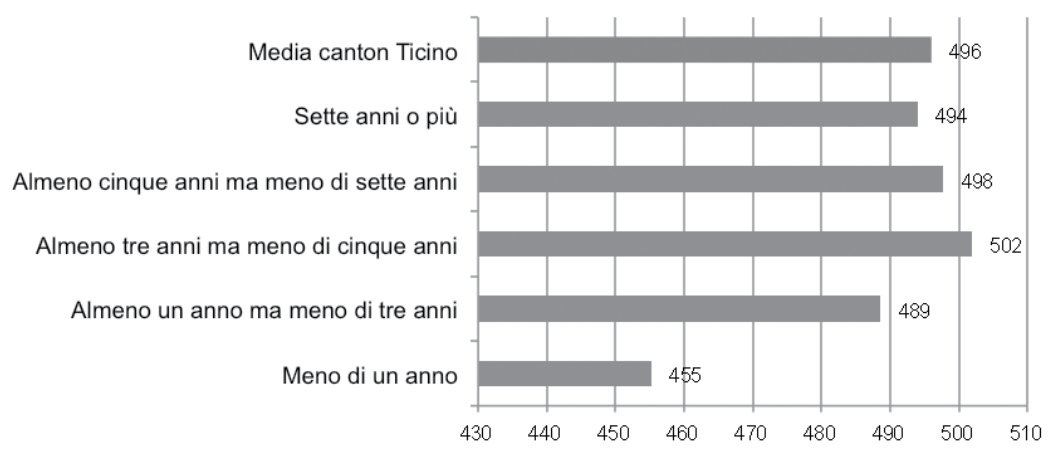

Figura 4: Punteggio medio ICILS secondo l'età di inizio uso delle TIC

In primo luogo è importante porre l'attenzione sul fatto che non vi è una correlazione forte tra l'età di inizio uso e le competenze in ICILS. Per questa ragione si è cercato un altro punto di confronto, mostrando semplicemente le medie ottenute dagli allievi a dipendenza dalla loro risposta alla domanda «da quanto tempo fai uso delle TIC?» (Figura 4). La differenza di punteggio tra chi usa le TIC da meno di un anno è molto ampia e statisticamente significativa in relazione agli allievi che le usano da più tempo: c'è una differenza di 47 punti con gli allievi che usano le TIC da almeno tre anni ma meno di cinque e comunque una differenza di 41 punti con la media generale del Canton Ticino. I risultati di questo grafico espongono però un elemento non da trascurare: gli allievi che usano dispositivi digitali da più tempo di tutti (da sette anni o più) non ottengono un punteggio più elevato degli altri; in effetti gli allievi che ottengono i risultati migliori sono quelli che usano le TIC da quando avevano 8-10 anni. Indubbiamente avere esperienza nell'uso delle TIC ha permesso di ottenere un punteggio più alto rispetto a chi si avvicina a questi strumenti da meno tempo. Tuttavia possiamo affermare che non c'è un aumento di prestazione all'aumentare del tempo di utilizzo, anzi, sembra che dopo un certo tempo ci sia un declino.

$\mathrm{Ci}$ si può interrogare quindi sul tipo e la frequenza di utilizzo che vengono fatti di questi strumenti, in particolare per gli utilizzi in giovanissima età (età prescolare o durante la scuola dell'infanzia o i primi anni delle elementari). Si potrebbe ipotizzare che gli allievi che hanno iniziato a usare le tecnologie quando avevano 10-12 anni le hanno incontrate in una fase di forte apprendimento e di scoperta, mentre chi le usava da più tempo ritiene magari di aver imparato a sufficienza, fermandosi a livelli inferiori. Questi elementi fanno quindi riflettere sul fatto che l'introduzione precoce all'uso di dispositivi digitali, sia a casa che a scuola, non sembra essere la strategia più adatta per permettere agli allievi di sviluppare le proprie competenze digitali. Si tratta di un risultato interessante, che, per analogia, richiama le conclusioni del rapporto OECD, Students, Computers and Learning (2015). 


\section{Tempo d'uso}

Un secondo tema analizzato è il tempo d'uso, con l'ipotesi che più tempo dedicato all'uso delle tecnologie digitali porti ad un maggiore sviluppo di competenze. Le domande legate al tempo di utilizzo del computer sono sostanzialmente due: la frequenza di utilizzo del computer e di internet a casa e a scuola.

Le frequenze di utilizzo a seconda del luogo in cui si trovano gli allievi sono molto differenti: il $43 \%$ afferma di trascorrere del tempo al computer tutti i giorni nel proprio tempo libero contro solo l' $1 \%$ che lo fa tutti i giorni a scuola. Una differenza simile la troviamo tra coloro che affermano di utilizzare il computer meno di una volta al mese: se ci si trova a scuola si tratta del $60 \%$ se invece si pensa a quando si è nella propria abitazione è il 5\% che lo afferma.

Le analisi successive (Tabella 5 e Figure 5 e 6) mostrano come queste differenze non influenzino le competenze: un massiccio utilizzo del computer a casa non permette da solo di migliorare le prestazioni, così come lo scarso utilizzo a scuola di per sé non le penalizza. La differenza tra il punteggio in ICILS di chi usa molto il computer a casa (502) e chi lo usa poco a scuola (484) è di 18 punti.

$\mathrm{Ci}$ si può quindi interrogare sulla tipologia d'uso effettuata del computer da parte degli adolescenti e come questa possa permettere di raggiungere competenze elevate in ICILS. L'uso del computer a casa è indubbio che permetta di ottenere prestazioni più alte rispetto a chi non lo usa del tutto o molto poco (48 punti in meno, rispettivamente 43). Mentre la differenza di punteggio tra chi a scuola lo usa molto e chi lo usa poco è piuttosto ridotta. Sembrerebbe dunque che il tipo di utilizzo effettuato dagli allievi, a casa e a scuola, non sia lo stesso e che l'utilizzo a casa sia infine più efficace in termine di acquisizione di competenze digitali e, soprattutto in ciò che viene poi richiesto esplicitamente nel test ICILS. Questo rilievo coincide con i risultati che presentiamo sotto sull'uso scolastico delle TIC, e anche con i risultati ottenuti dallo studio PISA (OECD, 2015), che sembrano indicare l'esistenza di una "giusta misura» di uso a scuola, che massimizza i risultati in termini di sviluppo delle competenze digitali.

\section{Tabella 5: Utilizzo del computer a casa e a scuola secondo il punteggio ICILS}

\begin{tabular}{lccccc} 
& Mai & $\begin{array}{c}\text { Meno di una } \\
\text { volta al mese }\end{array}$ & $\begin{array}{c}\text { Almeno una volta } \\
\text { al mese ma non } \\
\text { tutte le settimane }\end{array}$ & $\begin{array}{c}\text { Almeno una volta } \\
\text { alla settimana ma } \\
\text { non tutti i giorni }\end{array}$ & $\begin{array}{c}\text { Tutti i } \\
\text { giorni }\end{array}$ \\
\hline Uso domestico & 454 & 469 & 487 & 497 & 502 \\
Uso scolastico & 484 & 500 & 502 & 484 & 491 \\
\hline
\end{tabular}




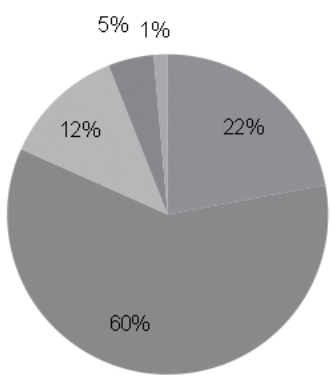

Figura 5: Utilizzo del computer a scuola

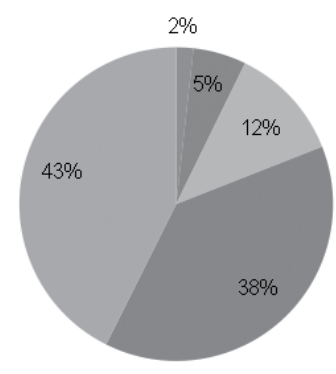

Meno di una volta al mese

Almeno una volta al mese ma non tutte le settimane

- Almeno una volta alla settimana ma non tutti i giorni

= Tutti i giorni

\section{Profilo d'uso}

Se dunque non sono queste variabili quantitative (età di primo uso; tempo d'uso a casa e a scuola) a incidere significativamente sullo sviluppo delle competenze digitali misurate da ICILS, potremo pensare che il tipo d'uso sia rilevante. I più recenti studi (Waller et al., 2016; Suter et al., 2015) mostrano infatti che l'uso delle TIC è oggi estremamente diversificato: usiamo lo stesso dispositivo per ascoltare musica, giocare ai videogiochi, elaborare immagini digitale, comunicare con gli amici, fare i compiti, ecc.

Diverse domande del questionario riguardavano la frequenza di utilizzo per attività specifiche, portando cioè lo sguardo dall' "usare il computer» al contenuto dell'azione: scrivere un testo per la scuola, inviare messaggi agli amici, leggere notizie sportive o per creare pagine web per i fan di una serie televisiva. Alcune domande chiedevano ad esempio quante volte si usavano le tecnologie per creare una presentazione oppure per creare degli articoli da inserire su un blog o un sito internet, per cercare delle informazioni su internet o per fare dei lavori scolastici. Le risposte a queste domande sono state classificate in quattro gruppi (Tabella 6 e Figura 7):

(a) Uso comunicativo, che riguarda tutte le attività in cui l'utente manda messaggi a uno o più altri utenti, ad esempio sui social networks, l'uso della mail, il commento ad articoli su blog o siti web;

(b) Uso informativo, che include le attività in cui l'utente cerca, seleziona e acquisisce informazioni online usando siti specifici, motori di ricerca, piattaforme, ecc.;

(c) Uso creativo/produttivo, che comprende tutte le attività nelle quali l'utente crea un prodotto originale, una presentazione, un documento, un video, ecc.;

(d) Uso scolastico, che include tutte le attività (eventualmente anche relative alle altre tre categorie) che vengono svolte in relazione in particolare alla scuola, quindi nel fare i compiti, preparare presentazioni, fare esercizi, ecc. 
Ogni attività elencata nella Tabella 7 in ciascun gruppo rappresentava, nel questionario ICILS una domanda con una scala in cui l'allievo doveva indicare quanto eseguiva quella specifica attività: mai, meno di una volta al mese, una volta al mese ma non tutte le settimane ecc. fino a tutti i giorni. Si è quindi trattato di raggruppare queste risposte in un unico indice per tipologia di utilizzo. Al fine di poter avere una classifica e capire il reale utilizzo per ragioni scolastiche, creative comunicative o informative il campione è stato suddiviso in tre gruppi equipollenti (33\%).

La Tabella 6 permette di individuare le medie ICILS ottenute dagli allievi in relazione all'utilizzo effettuato per i quattro gruppi di attività. Senza sorprese, possiamo notare innanzitutto che, a un uso scarso in tutti i gruppi, corrispondono punteggi ICILS piuttosto bassi.

Tabella 6: Attività per gruppi e relativi punteggi

\begin{tabular}{|c|c|c|c|c|}
\hline \multirow[b]{2}{*}{ Gruppo } & \multirow[b]{2}{*}{ Attività } & \multicolumn{3}{|c|}{$\begin{array}{l}\text { Modalità d'uso e punteggio } \\
\text { medio ICILS }\end{array}$} \\
\hline & & $\begin{array}{l}\text { Uso poco } \\
\text { frequente }\end{array}$ & $\begin{array}{c}\text { Uso } \\
\text { media- } \\
\text { mente } \\
\text { frequente }\end{array}$ & $\begin{array}{c}\text { Uso } \\
\text { molto } \\
\text { frequente }\end{array}$ \\
\hline $\begin{array}{l}\text { Comuni- } \\
\text { cativo }\end{array}$ & $\begin{array}{l}\text { - Comunicare sui social network } \\
\text { - Postare dei commenti sotto i vari profili o sui } \\
\text { blog } \\
\text { - Rispondere alle domande altrui sui forum } \\
\text { - Scrivere post sul proprio blog } \\
\text { - Caricare immagini o video }\end{array}$ & 489 & 497 & 501 \\
\hline $\begin{array}{l}\text { Infor- } \\
\text { mativo }\end{array}$ & $\begin{array}{l}\text { - Usare un software a fini didattici } \\
\text { - Cercare informazioni per studio o lavoro } \\
\text { scolastico } \\
\text { - Accedere a Wikipedia o un'enciclopedia in rete } \\
\text { - Porre domande in un forum o su una pagina } \\
\text { internet } \\
\text { - Trovare di posti in rete } \\
\text { - Leggere delle recensioni } \\
\text { - Trovare delle notizie } \\
\text { - Guardare video }\end{array}$ & 481 & 502 & 501 \\
\hline $\begin{array}{l}\text { Creativo/ } \\
\text { produttivo }\end{array}$ & $\begin{array}{l}\text { - Creare o modificare dei documenti } \\
\text { - Creare una semplice presentazione con diapo- } \\
\text { sitive } \\
\text { - Creare una presentazione multimedia } \\
\text { - Scrivere programmi per il computer } \\
\text { - Usare programmi di grafica } \\
\text { - Costruire o modificare una pagina internet } \\
\text { - Usare fogli di calcolo }\end{array}$ & 489 & 500 & 501 \\
\hline $\begin{array}{l}\text { Uso } \\
\text { scolastico }\end{array}$ & $\begin{array}{l}\text { - Redigere lavori scritti } \\
\text { - Preparare presentazioni } \\
\text { - Completare fogli di lavoro } \\
\text { - Organizzare il proprio tempo e il proprio } \\
\text { lavoro } \\
\text { - Scrivere in relazione all'apprendimento } \\
\text { - Fare delle verifiche }\end{array}$ & 488 & 507 & 496 \\
\hline
\end{tabular}




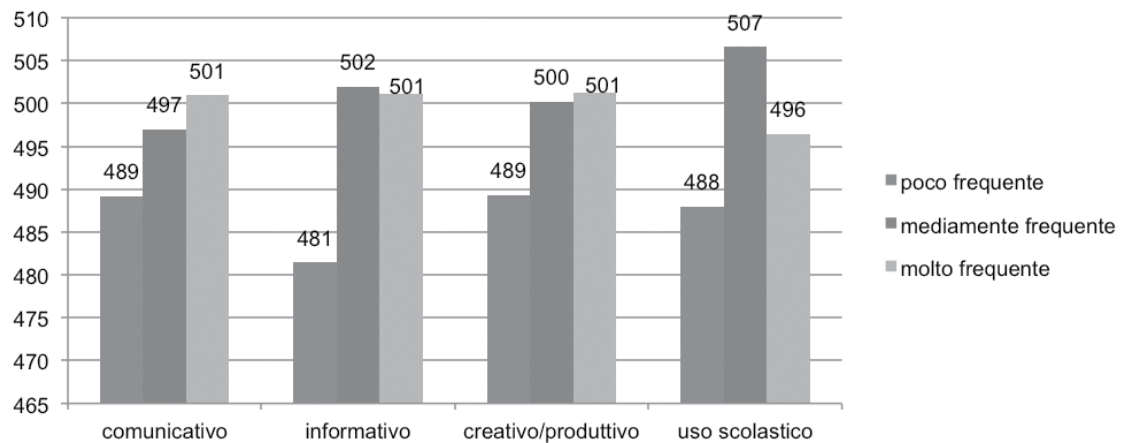

Figura 7: Media ICILS in relazione alla frequenza di utilizzo per diverse attività

Per due di essi (comunicativo e creativo) la tendenza è proporzionale: più l'uso è frequente più il punteggio aumenta. Lo scarso utilizzo a scopo informativo mostra una diminuzione del punteggio rispetto agli altri e l'uso per scopi scolastici è migliore se vengono utilizzati mediamente di frequente. Anche in questo caso sono state svolte delle correlazioni tra la media ICILS e la media di ciascuna tipologia d'uso ma non vi sono relazioni di rilievo, anche se le differenze di punteggio tra chi usa poco e chi usa molto sono statisticamente significative per le prime tre tipologie del grafico. Per quanto riguarda l'uso legato alla scuola sono le differenze tra mediamente usato e le altre due scale ad essere statisticamente significative.

Osservando la tipologia e le frequenze di utilizzo, in particolar modo a casa, si nota che il maggiore utilizzo svolto a casa riguarda le attività di tipo comunicativo e informativo (confermando così il profilo rilevato anche dallo studio JAMES; Waller et al., 2016): l' $86 \%$ che utilizza il computer tutti i giorni a casa lo fa con questo scopo, raggruppando gli allievi che lo fanno mediamente di frequente e molto di frequente. Troviamo poi anche coloro che vanno alla ricerca di informazioni: raggruppando chi lo fa mediamente spesso e spesso trovando un $84 \%$. Interessante notare che tra chi usa il computer tutti i giorni a casa vi è un $63 \%$ che lo fa per la scuola, tra mediamente frequente e molto frequente.

L'interpretazione dei risultati relativi all'uso scolastico solleva alcune domande. Si tratta infatti dell'unico gruppo di attività nel quale un uso più frequente porta a una diminuzione della competenza. Una possibile ipotesi è che gli studenti che dichiarano un uso «mediamente frequente», ne facciano un uso mirato, relativo al compito che si sta svolgendo (una traduzione, un problema di matematica, la scrittura di un testo, ecc.). Chi invece dichiara un uso «molto frequente» ne fa invece un uso di accompagnamento, cioè di attività parallela allo studio (ascoltare musica, inviare messaggi, consultare notizie, ecc.) - quindi con attività che sono antagoniste alla concentrazione sul lavoro di apprendimento. Un'altra variabile, non misurata in questo studio, che sicuramente influisce su 
questi dati è l'uso che il docente propone in classe per la sua disciplina, e in particolare se il docente ha suggerito o proposto una modalità specifica di uso di apprendimento delle tecnologie. Questa ulteriore interpretazione è allineata con quanto analizzato sopra a proposito del tempo d'uso a scuola, nonché con le conclusioni esposte in OECD (2015).

\section{Conclusion $i$}

A partire dalla constatazione della diffusione e rilevanza delle tecnologie digitali nella scuola e nel tempo libero, questo studio si è interrogato sullo sviluppo delle competenze digitali nei giovani. Sfruttando i dati raccolti dallo studio ICILS, sono state proposte delle analisi con l'obiettivo di capire l'influenza dell'età del primo utilizzo, del tempo di utilizzo e della tipologia di attività delle nuove tecnologie sulle competenze misurate poi nel test ICILS. I dati si riferiscono unicamente al Canton Ticino, i cui raccolti da ICILS 2013 sono rappresentativi della popolazione di allievi di tredici anni, permettendo un confronto con gli altri paesi partecipanti. L'analisi ha usato strumenti descrittivi, analizzando le relazioni tra i dati del questionario rivolto agli allievi dopo la somministrazione del test.

I risultati dell'indagine ICILS 2013, nel confronto tra gli Stati che hanno partecipato all'indagine, indicano che gli allievi ticinesi hanno ottenuto un punteggio medio di 496 punti, non discostandosi in maniera statisticamente significativa dalla media internazionale, che era fissata a 500 punti.

In termini assoluti, questo risultato si pone prossimo al limite inferiore del secondo livello di competenza su una scala di quattro. Concretamente, una persona che raggiunge tale livello è in grado di svolgere autonomamente semplici attività che richiedono l'uso delle TIC, ma deve essere supportato per trattare compiti più complessi; ha consapevolezza del diverso grado di affidabilità delle informazioni presenti sul web, ma non possiede appieno gli strumenti per selezionarle; sa che esistono dei pericoli a esporre sulla rete informazioni personali, ma non è sempre capace di riconoscerli; e ha, infine, solo scarse cognizioni dei diritti intellettuali legati a ciò che si trova su internet (Fraillon et al., 2014).

In termini relativi, i risultati ticinesi destano qualche preoccupazione, poiché ben dieci Stati su quattrodici hanno ottenuto punteggi significativamente superiori alla media. Va inoltre tenuto conto del fatto che la media internazionale è fortemente condizionata in negativo dai risultati ottenuti in Tailandia e Turchia, nettamente inferiori a quelli degli altri paesi. Occorre infine sottolineare che, sebbene nessun risultato medio nazionale si situi al disopra del livello 2 , in cinque Stati esso è più prossimo al livello superiore di quanto non lo sia a quello inferiore.

Le principali ipotesi sulle ragioni del risultato relativamente modesto conseguito dagli studenti ticinesi ci giungono dal grado di strutturazione della formazione alle nuove tecnologie nel sistema cantonale. Gli Stati che hanno ottenuto i 
risultati migliori - Repubblica Ceca, Australia e Polonia - sono accomunati dal fatto che la politica scolastica in questo ambito, oltre che a livello regionale, è definita, in maniera più marcata rispetto a quanto avviene in Svizzera, anche a livello nazionale. Un fatto ancora più rilevante in relazione a un ambito, quale è quello delle nuove tecnologie, che necessita anche di investimenti importanti. In questi paesi, inoltre, l'insegnamento di una materia specificamente legata alle TIC è previsto, obbligatorio e, quanto meno all'ottavo anno di scolarità, valutato. In Ticino non esiste una disciplina obbligatoria che preveda una valutazione di queste competenze e il livello decisionale - e, presumibilmente, il finanziamento - è prevalentemente cantonale.

Questi risultati evidenziano come, in assenza di una chiara politica di integrazione delle TIC nell'insegnamento, i giovani siano tuttavia in grado di sviluppare autonomamente competenze discrete fuori dalle aule scolastiche, anche se senza raggiungere livelli elevati. Da un lato, questo permette di prendere le distanze dall'idea che i «nativi digitali» siano naturalmente predisposti allo sviluppo di competenze digitali superiori. Dall'altro, ciò potrebbe suggerire che una politica educativa timida in questo ambito - come l'identificazione dell'alfabetizzazione informatica e comunicazione come una competenza "trasversale», che non necessita la sua integrazione in una specifica disciplina - potrebbe essere poco incisiva, incidendo in maniera minima in un processo che ha radici e prende forma in un contesto informale. Dal punto di vista dell'educazione ai media e a un uso sano, legale e sicuro delle tecnologie, questo approccio presenta l'ulteriore svantaggio di non creare un luogo di incontro esplicito tra le pratiche digitali dei giovani e degli adulti.

Abbiamo dunque considerato i dati ticinesi come relativi ad un apprendimento informale e non scolastico di competenze tecnologiche. L'analisi ha rivelato diverse indicazioni che riteniamo utili per pensare un percorso coerente ed efficace di sviluppo della computer and information literacy. Innanzitutto, i risultati mostrano come l'età di primo uso delle tecnologie sia rilevante, anche se non nel senso di "prima è meglio»: si raggiungono buoni livelli di competenza se si incontrano le tecnologie digitali a partire dal II ciclo di scuola elementare, mentre un uso precoce sembra in realtà bloccare lo sviluppo di competenze di alto livello.

Usare molto le tecnologie durante l'età dell'adolescenza (tanto a scuola quanto fuori) non porta a maggiore competenza, anche se un uso scarso e saltuario ne limita lo sviluppo. Una conclusione simile, per analogia, si ritrova in (OECD, 2015). L'identificazione della giusta misura è una sfida che, verosimilmente, risponde a parametri locali e complessi.

Abbiamo infine messo in luce che l'aumento dell'uso scolastico delle tecnologie digitali segue un pattern diverso in relazione allo sviluppo delle competenze di literacy. Questo dato è interessante, perché potrebbe indicare che sotto l'etichetta di «uso delle tecnologie per fare i compiti» si nascondano due diversi tipi d'uso: un uso relativo al contenuto dello studio (efficace), e un altro parallelo (e 
distraente). In questo senso, come richiama anche OCSE (2015), diventa ancora più centrale il ruolo dei docenti, che possono mostrare e proporre validi metodi d'integrazione delle tecnologie nello studio. Dal punto di vista della ricerca, questo risultato invita a un'analisi più approfondita e qualitativa delle pratiche digitale dei giovani, andando oltre la pura quantificazione dell'uso nei diversi contesti.

\section{Annotazione}

1 Si noti che la Norvegia ha fatto partecipare i suoi allievi del nono anno scolastico, mentre tutti gli altri quelli dell'ottavo.

\section{Riferimenti bibliografic i}

Ainley, J., Enger, L. \& Searle D. (2008). Students in a digital age: Implications of ICT for teaching and learning. In J. Voogt \& G. Knezek G. (Éd.), International handbook of information technology in primary and secondary education (pp. 63-80). Berlin: Springer. doi: 10.1007/978-0-387-73315-9_4

Audunson, R. \& Nordlie, R. (2003). Information literacy: the case or non-case of Norway? Library Review, 52, (7), 319-325.

BAKOM. (2016). Strategie Digitale Schweiz. Bern: Confederazione Svizzera.

Bottani, N. (2015),.Un'ardua ascesa non priva di tornanti: le vicende delle indagini internazionali su vasta scala. In CIRSE (Éd.), Prove standardizzate in ambito educativo: confronto tra Svizzera e Italia. Atti del convegno CHI-I (pp.15-16). Locarno: CIRSE.

Botturi, L. (in stampa). When the big picture is not enough. Media Education Research Journal, 8, (1).

Bullen, M., Morgan, T., Belfer, K. \& Qayyum, A. (2009). The Net Generation in Higher Education: Rhetoric and Reality. International Journal of Excellence in E-Learning, 2, (1), 1-13. Retrieved from https://journals.hbmsu.ac.ae/Pages/Articles.aspx?AID=91\&IID=21

Bullen, M., Morgan, T. \& Qayyum, A. (2011). Digital Learners in Higher Education: Generation is Not the Issue. Canadian Journal of Learning Technology, 37, (1), 1-24. doi: $10.21432 / \mathrm{T} 2 \mathrm{NC} 7 \mathrm{~B}$

Calvo, S. \& Zampieri, S., (2017). ICILS 2013. Come comunicano gli adolescenti con le nuove tecnologie. Locarno: Centro innovazione e ricerca sui sistemi educativi.

Camerini, A. L., Schulz, P. J. \& Jeannet, A. M. (2017). The social inequalities of internet access, its use and the impact on children's academic performance: Evidence from a longitudinal study in Switzerland. New Media \& Society. doi:10.1177/1461444817725918

DECS. (2004). Piano di formazione della scuola media. Bellinzona: Dipartimento dell'educazione, della cultura e dello sport, Divisione della scuola.

DECS. (2015a). Piano degli studi della scuola dell'obbligo. Bellinzona: Dipartimento dell'educazione, della cultura e dello sport, Divisione della scuola.

DECS. (2015b). La scuola che verrà. Bellinzona: Dipartimento dell'educazione, della cultura e dello sport, Divisione della scuola.

Eickelmann, B., Bos, W., Gerick, J. \& Kahnert, J. (2014). Anlage, Durchführung und Instrumentierung von ICILS 2013. In W. Bos, B. Eickelmann, J. Gerick, F. Goldhammer, H. Schaumburg, K. Schwippert, M. Senkbeil, Martin, R. Schulz-Zander \& H. Wendt (Éd.), ICILS 2013. Computer- und informationsbezogene Kompetenzen von Schülerinnen und Schülern in der 8. Jahrgangsstufe im internationalen Vergleich (pp. 33-42). Münster: Waxmann.

Erstad, O. (2004). PILOTer for skoleutvikling (PILOTs for school development) (Research UniPub Report no. 28, TD43 (1)). ITU, University of Oslo. 
Fraillon, J., Ainley, J., Schulz, W., Friedman, T. \& Gebhardt, E. (2014). Preparing for Life in a Digital Age. The IEA International Computer and Information Literacy Study International Report. Springer Open. Retrieved from http://www.iea.nl/fileadmin/user_upload/Publications/Electronic_versions/ICILS_2013_International_Report.pdf

Fraillon, J., Schulz, W. \& Ainley, J. (2013). International Computer and Information Literacy Study. Assessment framework. Amsterdam: IEA.

Giovani e Media. (2016). Competenze mediali. Berna: Ufficio federale delle assicurazioni sociali.

Gruppo di lavoro e-education. (2012). Rapporto conclusivo e-education. Bellinzona: Dipartimento dell'educazione, della cultura e dello sport.

Helsper, E. J. \& Eynon, R. (2009). Digital natives: where is the evidence? British Educational Research Journal, 36, (3), 503-520.

Hobbs, R. (2010). Digital and Media Literacy. A Plan of Action. Washington, DC: Aspen Institute. Retrieved from https://www.knightfoundation.org/media/uploads/publication_ pdfs/Digital_and_Media_Literacy_A_Plan_of_Action.pdf

Jones, C. (2010). A new generation of learners? The Net Generation and Digital Natives [Special issue on Learning, the Net Generation and Digital Natives]. Learning, Media and Technology, 35, (4), 365-368.

Livingstone, S. \& Sefton-Green, J. (2016). The Class. Living and Learning in the Digital Age. New York, NY: University Press.

Lorenz, R., Gerick, J., Schulz-Zander, R. \& Eickelmann, B. (2014). Computer- und informationsbezogene Kompetenzen von Mädchen und Jungen im internationalen Vergleich. In W. Bos, B. Eickelmann, J. Gerick, F. Goldhammer, H. Schaumburg, K. Schwippert, M. Senkbeil, Martin, R. Schulz-Zander \& H. Wendt (Éd.), ICILS 2013. Computer-und informationsbezogene Kompetenzen von Schülerinnen und Schülern in der 8. Jahrgangsstufe im internationalen Vergleich (pp. 231-264). Münster: Waxmann.

O’Reilly, T. (2005). What is Web 2.0? In H. Donelan, K. Kear \& M. Ramage (Éd.), Online Communication and Collaboration: A reader (pp. 225-235). Abingdon: Routledge.

OECD. (2015). Students, Computers and Learning: Making the Connection. PISA, OECD Publishing. Retrieved from http://www.oecd.org/publications/students-computers-and-learning-9789264239555-en.htm

Palfrey, J. \& Gasser, U. (2008). Born digital: Understanding the first generation of Digital Natives. New York, NY: Basic Books.

Prensky, M. (2001a). Digital Natives, Digital Immigrants. On the Horizon, 9, (5), 1-6. doi:10.1108/10748120110424816

Prensky, M. (2001b). Digital Natives, Digital Immigrants, Part II; Do They Really Think Differently? On the Horizon, 9, (6), 1-6. doi:10.1108/10748120110424843

Salvisberg, M. \& Zampieri, S. (2015). Valutazioni sotto esame. Piste esplorative per un confronto tra PISA e note scolastiche 2009 e 2012. Locarno: Centro Innovazione e Ricerca sui Sistemi Educativi, SUPSI.

Schulmeister, R. (2008). Is there a net generation in the house? Dispelling a mystification. ELeEd - eLearning and Education Journal (Issue 5). Retrieved from https://eleed.campussource.de/archive/5/1587

SEFRI. (2017). Herausforderungen der Digitalisierung für Bildung und Forschung in der Schweiz. Bern: Confederazione Svizzera.

Selwyn, N. (2006). Exploring the digital disconnect between net savvy students and their schools. Learning, Media and Technology, 31, (1), 5-17.

Senkbeil, M., Goldhammer, F., Bos, W., Eickelmann, B., Schwippert, K. \& Gerick, J. (2014). Das Konstrukt der computer- und informationsbezogenen Kompetenzen in ICILS 2013. In W. Bos, B. Eickelmann, J. Gerick, F. Goldhammer, H. Schaumburg, K. Schwippert, M. Senkbeil, Martin, R. Schulz-Zander \& H. Wendt (Éd.), ICILS 2013. Computer- und informationsbezogene Kompetenzen von Schülerinnen und Schülern in der 8. Jahrgangsstufe 
im internationalen Vergleich (pp. 83-112). Münster: Waxmann.

Suter, L., Waller, G., Genner, S., Oppliger, S., Willemse, I., Schwarz, B. \& Süss, D. (2015). MIKE - Medien, Interaktion, Kinder, Eltern. Zürich: Zürcher Hochschule für Angewandte Wissenschaften.

Tapscott, D. (1998). Growing up digital: The rise of the Net Generation. New York, NY: McGraw-Hill.

Teo, T. (2013) An initial development and validation of a Digital Natives Assessment Scale (DNAS). Computers \& Education, 67, 51-57.

Teo, T. (2016). Do digital natives differ by computer self-efficacy and experience? An empirical study. Interactive Learning Environments, 24, (7), 1725-1739.

Thompson, P. (2013). The digital natives as learners: technology use patterns and approaches to learning. Computers \& Education, 65, 12-33.

Waller, G., Willemse, I., Genner, S., Suter L. \& Süss, D. (2016). JAMES - Gioventu', attivita , media - rilevamento Svizzera. Zurigo: Zürcher Hochschule für Angewandte Wissenschaften.

Wang, S.-K., Hsu, H.-Y., Campbell, T. Coster, D. C. \& Longhurst, M. (2014). An investigation of middle school science teachers and students use of technology inside and outside of classrooms: considering whether digital natives are more technology savvy than their teachers. Educational Technology Research and Development, 62, (6), 637-662.

Wendt, H., Vennemann, M., Schwippert, K. \& Drossel, K. (2014). Soziale Herkunft und computer- und informationsbezogene Kompetenzen von Schülerinnen und Schülern im internationalen Vergleich. In W. Bos, B. Eickelmann, J. Gerick, F. Goldhammer, H. Schaumburg, K. Schwippert, M. enkbeil, Martin, R. Schulz-Zander \& H. Wendt (Éd.), ICILS 2013. Computer- und informationsbezogene Kompetenzen von Schülerinnen und Schülern in der 8. Jahrgangsstufe im internationalen Vergleich (pp. 265- 296). Münster: Waxmann.

Yong, S. T. \& Gates, P. (2014). Born Digital: Are They Really Digital Natives? International Journal of e-Education, e-Business, e-Management and e-Learning, 4, (2), 102-105.

Parole chiave: Competenze digitali, apprendimento informale, nativi digitali, media education. 


\section{Jugendliche und IT-Kompetenzen: Zwischen Digital Natives und wirksamen Fertigkeiten}

\section{Zusammenfassung}

Generationsbeschreibungen zum Umgang mit digitalen Technologien wurden seit Jahren vom Konzept so genannter "digital natives” geprägt. Im Gegensatz dazu wurden etwas ältere Menschen der vorangehenden Generationen als "digital immigrants" bezeichnet. Auch wenn verschiedene Studien solche Zuschreibungen kritisieren, hält sich diese Differenzierung in gegenwärtigen Diskussion. Die ICIL-Studie bietet relevante Daten, um das Verhältnis von Heranwachsenden und ihrem Umgang mit neuen medialen Technologien besser verstehen zu können. Unsere Analyse fokussiert auf ein geographisch bzw. soziostrukturell ausgewähltes Gebiet, wo die schulischen Institutionen bisher keine Leitungsrolle übernommen haben. In diesem Kontext versucht die vorliegende Untersuchung, folgende Fragen zu beantworten: Welche Eigenschaften des Schulsystems fördern die Entwicklung von Kompetenzen im Bereich Medien und Informatik? Lassen sich zwischen dem Alter der Erstnutzung, der Nutzungsfrequenz, der Art der Nutzung von digitalen Medien, und den digitalen Kompetenzen von Lernenden Zusammenhänge finden? Die Diskussion versucht Überlegungen zur Rolle der Schule und der Schulpolitik zur Nutzung von digitalen Geräten und dem Internet durch Jugendliche darzulegen.

Schlagworte: Digital literacy, informelles Lernen, digital natives, Medienpädagogik

\section{Jeunes et technologies: entre natifs numériques et compétences réelles}

\section{Ré sumé}

La comparaison entre générations dans le domaine des technologies est caractérisée notamment par la définition des «natifs numériques» qui met en contraste les nouvelles générations avec celles plus anciennes définies comme «immigrés numériques». Différentes études mettent en cause ces enjeux. L'étude ICILS met à disposition des données robustes pour se pencher sur le rapport que les jeunes entretiennent avec les technologies, dans un contexte géographique et social où les institutions éducatives n'ont pas encore assumé un rôle défini. Cette étude essaie de fournir des réponses aux questions suivantes: quelles caractéristiques des institutions éducatives favorisent-elles le développement des compétences numériques? Existe-t-elle une relation entre les compétences numériques et l'âge de la première utilisation des technologies, la fréquence et le type d'utilisation? Pour conclure, on essaie d'ouvrir la réflexion sur le rôle de l'école et des politiques éducatives dans l'utilisation des dispositifs digitaux et d'internet par les élèves. 
Mots-clés: Compétences numériques, apprentissage informel, natifs numériques, éducation aux médias

\section{Young People and Technologies: Between Digital Natives and Actual Competences}

\section{Summary}

The discussion about the digital generation gap has been lately dominated by the concept of "digital natives», which characterises the younger generation against older «digital immigrants». Different research studies counter such claim, or at least put it in a critical perspective, but the debate is still ongoing. The ICILS study offers sound data in order to analyse the relationship between young people and technologies, in particular within the borders of a delimited geographic and social setting in which schools have not yet acquired a central role in the digital transformation. This paper addresses the following questions: what features of the education system promote the development of digital competences? Can we establish a relationship between age of first use, frequency of use and type of use of digital technologies, and the development of digital competences in different domains? The discussion offers elements to reflect on the role of school systems and policies in young people's use of digital devices and the Internet.

Keywords: Digital literacy, informal learning, digital natives, media education 
